\title{
ORIGINAL
}

\section{The new procedure for manual CPAP titration : the afternoon CPAP titration (aPT)}

\author{
Keisuke Kido ${ }^{1,2,3}$ and Naoko Tachibana ${ }^{1,2,3}$ \\ ${ }^{1}$ Division of Sleep Medicine, Kansai Electric Power Medical Research Institute, Osaka, Japan, ${ }^{2}$ Center for Sleep-related Disorders, Kansai \\ Electric Power Hospital, Osaka, Japan, ${ }^{3}$ Department of Sleep Medicine, Division of Health Science, Graduate School of Medicine, Osaka \\ University, Osaka, Japan
}

\begin{abstract}
Study Objectives : Although the full-night continuous positive airway pressure (CPAP) titration (fnPT) has been recognized as the gold standard for determining an optimal therapeutic pressure for obstructive sleep apnea (OSA) treatment, it is labor-intensive, time-consuming because it requires overnight polysomnography attended by well-experienced sleep technologists. The aim of this study is to develop a practical and feasible alternative titration method. Methods : We assessed demographic data and diagnostic polysomnographic parameters, time spent in CPAP titration, CPAP efficacy and long-term adherence of the two groups of our OSA patients who had received CPAP titration either by fnPT $(n=46)$ or by afternoon CPAP titration $(a P T, n=22)$. Main results : Mean total recording time of aPT was significantly shorter than that by fnPT $(p<0.0001)$. There was no significant difference in mean residual apnea hypopnea index (AHI) on treatment, percent days with device usage, cumulative device usage hours during the 360 days, average device usage hours per day (360 days, days used), and percent of days with device usage $\geq 4$ hours during the 360 days after starting CPAP treatment between the two groups. Conclusions: Our study demonstrated that aPT was feasible procedure as an alternative to fnPT. J. Med. Invest. 68:170-174, February, 2021
\end{abstract}

Keywords : obstructive sleep apnea, polysomnography, CPAP titration, CPAP adherence

\section{INTRODUCTION}

Obstructive sleep apnea (OSA) is characterized by repetitive collapse of the upper airway during sleep, leading to recurrent arousals, intermittent hypoxia and hypercapnia and numerous surges of sympathetic activity $(1,2)$. It has been widely recognized that OSA is associated with neuropsychological dysfunction (3), decreased quality of life (4) and motor vehicle accidents (5). In addition, increased risk of hypertension (6), metabolic syndrome (7) and higher risk of cardiovascular diseases (8) have been well documented. In respect of the prevalence, OSA is high in the general adult population, ranging from 9 to $38 \%$ (9), which means that OSA is the one of the biggest public health problems.

As countermeasures, continuous positive airway pressure (CPAP) has been considered the first line treatment for OSA. CPAP improves quality of life (10), reduces motor vehicle accidents (11), decreases the risk of hypertension (12), metabolic syndrome (13) and cardiovascular diseases (14). Despite these highly significant treatment effects, CPAP adherence remains a major obstacle to its effectiveness (15). Although CPAP regular use is defined as at least 4 hours use on $70 \%$ of days (16), CPAP adherence remains highly variable (17-20). In the recent large randomized clinical trials (21), CPAP effects on hypertension and cardiovascular events were not significant, but the subgroup analysis of patients with OSA using CPAP for more than 4 hours per night demonstrated the significant treatment effects (22). This result alerted us to keep in mind that higher CPAP adherence is essential for OSA treatment.

Received for publication September 25, 2020 ; accepted February 10, 2021.

Address correspondence and reprint requests to Keisuke Kido, Center for Sleep-related Disorders, Kansai Electric Power Hospital 2-1-7, Fukushima, Fukushima, Osaka, 553-0003 JAPAN.
In this respect, various factors that could influence CPAP adherence have been studied in the western countries. The most recent comprehensive review by Mehrtash and his colleagues, summarized these factors from four different domains : socio-demographic characteristics, disease severity, psychosocial factors, and side-effects (23). In addition, it has been widely known that there is an optimal pressure of CPAP to stabilize the opening of the upper airway, which differs from one patient to another, so CPAP titration played an important role in increasing CPAP adherence (24). Consequently, American Academy of Sleep Medicine (AASM) established the clinical guideline (25) regulating that the standard way of CPAP titration should be conducted by the full-night CPAP titration (fnPT). The main aim of fnPT is to determine an optimal therapeutic pressure that abolishes respiratory events during sleep of OSA patients by using polysomnography (PSG) and a CPAP device at the same time. The drawback of fnPT is that this procedure requires overnight PSG at a sleep lab attended by well-experienced sleep technologists, resulting in high cost from the viewpoint of time and labor. Therefore, we need a practical alternative way of titration which is feasible in Japan where sleep medicine is still underdeveloped.

The aim of this study is to develop a practical and feasible alternative titration method, named afternoon CPAP titration (aPT).

\section{METHODS}

Patients

This is the retrospective study dealing with consecutive 122 OSA patients who underwent CPAP titration from April 2013 to March 2018 in our sleep laboratory (Figure 1). Our sleep center specialized in all kinds of sleep-related disorders, and it was our daily clinical routine to get demographic data such as gender, age, height, weight, and working status as well as Epworth 
sleepiness scale (ESS) at the first visits of new patients. When it was clinically indicated, attended all-night PSG for diagnosis was routinely performed. All the PSG data were scored according to AASM criteria (26). OSA was defined when apnea hypopnea index (AHI) was five or more on diagnostic PSG.

This study was approved by the Institutional Review Board of Kansai Electric Power Medical Research Institute. The study was conducted in according to the principles of the Declaration of Helsinki.

\section{CPAP education}

All the 122 patients received CPAP education lasting about 30 minutes as clinical routine of our sleep lab on the same day of the titration. This educational session was carried out by experienced sleep technologists before starting CPAP titration. The session was composed of explaining reasons for CPAP use, its clinical benefit, mask fitting including patients trialing ones of different styles, training of how to put on and take off a mask, hands-on demonstration of CPAP devices, and acclimatization to CPAP.

\section{CPAP titration}

FnPT was carried out by well-experienced sleep technologists using the AASM guidelines (25). As for fnPT, the optimal pressure setting procedure was based on the AASM algorithm (25). We invented aPT that was performed in the early afternoon starting around 2 p.m. and ending before 4 p.m. by using PSG when the patients was encouraged to take a nap. The procedure to find out an optimal pressure was mostly equivalent to fnPT except that fnPT required the occurrence of REM sleep on supine with no respiratory events or arousals. In addition, if the patients did not achieve REM sleep during aPT, the study was ended. We calculated the number of patients who achieved REM sleep during both aPT and fnPT.

Of the 122 patients, 72 patients underwent fnPT and 50 patients received aPT (Figure 1). The total recording time of fnPT and aPT was calculated after titration. Due to retrospective nature of this study, our patients were not randomly allocated to aPT or fnPT.

\section{CPAP prescription}

After the titration, sleep specialists reviewed the titration data. As auto-titrating CPAP devices are available to all the patients under the Japanese national medical insurance system, an optimal pressure range (for example, 4-7 $\mathrm{cmH}_{2} \mathrm{O}$ ) was prescribed except one patient who opted out to fixed-pressure. 117 out of 122 patients were given either REMStar (Philips Respironics, Murrysville, PA, USA) or DraemStation (Philips Respironics, Murrysville, PA, USA) and used the same machine throughout the study. As this is the retrospective study, these two types CPAP machines were not randomly allocated, however, the algorithm of these two machines from a single manufacturer, Philips Respironics, was basically identical. Five remaining patients who opted out to another CPAP machines from other manufactures than Philips Respironics were excluded at this point (Figure 1).

\section{CPAP follow-up system}

Regular CPAP follow-up at the clinic was mandatory according to the Japanese national medical insurance system. Patients were not allowed to purchase CPAP devices directly from the providers in Japan, but the public insurance completely covered the rental fee of CPAP devices on the condition that the patients come to the clinic at least once per three months. On their regular visits, the patients got CPAP adherence data downloaded by sleep technologists in addition to consultation by sleep specialists. Troubleshooting about CPAP devices or interfaces was sorted out by sleep technologists.

During the one year follow-up, the 49 patients were excluded for the following reasons : Patients who were unable to continue to use CPAP for more than one year (fnPT : 9, aPT : 21), patients who moved to another hospitals or sleep clinics (fnPT : 12, aPT : 6). One patient who underwent fnPT was excluded because his first one month data was missing due to technical error. As a results, the subjects for analysis were 46 patients after fnPT and 22 patients after aPT (Figure 1). As for the patients who were not able to continue to use CPAP for more than one year, we calculated CPAP dropout rate within one year.

\section{CPAP efficacy}

The CPAP device had capacity to record the nightly amount of the usage and to measure the airflow of users' breathing, enabling us to obtain the residual numbers of apnea and hypopneas. By downloading the data, mean residual AHI was available. As the CPAP device was unable to measure sleep time, the mean residual AHI was calculated by dividing the number of apneas and hypopneas by operating hours, however, this mean residual AHI has been widely used as CPAP efficacy.

\section{CPAP adherence}

We collected the data via a memory card at 360 days after the introduction of CPAP. This assessment included percent days with device usage during the 360 days, cumulative device usage

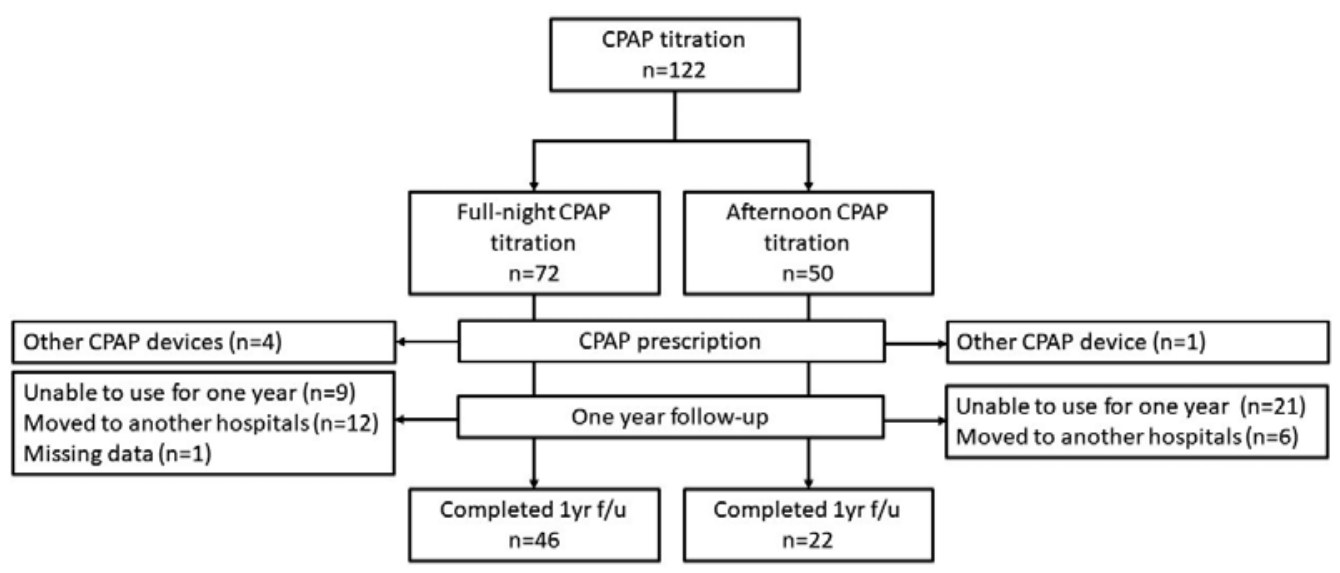

Figure 1. Design of the study. 
hours during the 360 days, average device usage hours per day during the 360 days, average device usage hours per day during the days of device used of this period, and percent of days with the device usage hours $\geq 4$ hours during the 360 days.

\section{Statistical analysis}

Statistical analysis was done using JMP PRO 14.0.0 (SAS Institute Technology, U.S.). All demographic, polysomnographic variables, sleep parameters and CPAP adherence data were described using mean and standard deviation. A chi-square analysis with Fisher's exact test was performed to compare categorical data. The $p$ values $<0.05$ were assessed to represent statistical significance.

\section{RESULTS}

\section{Demographic and diagnostic polysomnographic data}

Demographic characteristics of the two groups did not show significant difference (Table 1). As for CPAP dropout rates, group of aPT $(42.0 \%)$ was significantly higher than that of fnPT (12.5\%). Most of polysomnographic parameters at the diagnosis, such as total sleep time, sleep efficiency, sleep latency, AHI, the number of $3 \%$ desaturations per one hour sleep (oxygen desaturation index by $3 \%$, ODI3\%), lowest oxygen saturation (lowest $\mathrm{SpO}_{2}$ ), and arousal index were not significantly different between two groups (Table 2).

Table 1. Demographic data of our participants.

\begin{tabular}{llll}
\hline & fnPT & aPT & $p$ value \\
\hline Number & 46 & 22 & - \\
M/F & $43 / 3$ & $21 / 1$ & $1 .^{*}$ \\
Age (years) & $58.3 \pm 12.8$ & $58.9 \pm 12.3$ & .753 \\
Height $(\mathrm{cm})$ & $168.9 \pm 7.0$ & $168.6 \pm 6.1$ & .613 \\
Weight $(\mathrm{kg})$ & $77.1 \pm 14.5$ & $77.7 \pm 17.8$ & .733 \\
BMI (kg/m $)$ & $26.9 \pm 4.2$ & $27.1 \pm 4.9$ & .906 \\
Working status & & & \\
(Full-time/Part-time/Retire/ & $(36 / 1 / 9 / 0)$ & $(16 / 2 / 3 / 1)$ & $.220^{*}$ \\
Homemaker) & & & \\
ESS & $10.1 \pm 5.4$ & $10.4 \pm 4.3$ & .457 \\
\hline
\end{tabular}

Data presented $\mathrm{n}$ or as mean \pm standard deviation. BMI, body mass index ; ESS, Epworth sleepiness scale. *Fisher's exact test

Table 2. Diagnostic polysomnographic parameters in the two groups.

\begin{tabular}{llll}
\hline & fnPT $(\mathrm{n}=46)$ & $\mathrm{aPT}(\mathrm{n}=22)$ & $p$ value \\
\hline TST (min) & $376.5 \pm 71.5$ & $392.7 \pm 75.2$ & .362 \\
Sleep efficiency (\%) & $82.1 \pm 11.3$ & $83.0 \pm 12.0$ & .684 \\
Sleep latency (min) & $22.0 \pm 20.4$ & $16.1 \pm 19.3$ & .067 \\
AHI & $38.7 \pm 15.8$ & $43.3 \pm 18.3$ & .268 \\
ODI3\% & $34.6 \pm 16.8$ & $40.0 \pm 18.7$ & .211 \\
Lowest $\mathrm{SpO}_{2}(\%)$ & $77 \pm 8$ & $73 \pm 13$ & .325 \\
\% time $\mathrm{SpO}_{2}<90 \%(\%)$ & $7.4 \pm 9.1$ & $17.3 \pm 18.9$ & $.028^{\#}$ \\
Arousal index & $34.2 \pm 13.1$ & $38.5 \pm 16.7$ & .274 \\
\hline
\end{tabular}

Data presented as mean \pm standard deviation. TST, total sleep time ; AHI, apnea hypopnea index ; ODI3\%, the number of $3 \%$ desaturations per one hour sleep ; $\%$ time $\mathrm{SpO} 2<90 \%$, percentage of total sleep time with oxygen saturation below $90 \%$. \#statistical significance.
There was one parameter that showed significant difference, which was percentage of total sleep time with oxygen saturation below $90 \%$ (\% time $\mathrm{SpO}_{2}<90 \%$ ). This percentage was significantly higher in aPT than in fnPT group (17.3 \pm 18.9 vs $7.4 \pm 9.1$, $\mathrm{p}<0.03)$.

\section{REM sleep occurrence and time spent on CPAP titration}

8 out of 22 patients (36.4\%) achieved REM sleep during aPT, while all of the 46 patients $(100 \%)$ during fnPT $(\mathrm{p}<0.0001)$. Mean total recording time was significantly shorter in aPT group than in fnPT group (73.0 \pm 13.9 vs $501.5 \pm 41.1 \mathrm{~min}, \mathrm{p}<0.0001)$.

\section{CPAP efficacy and adherence}

There was no significant difference in mean residual AHI during the 360 days (fnPT vs. aPT, $3.6 \pm 2.5$ vs. $3.1 \pm 1.2$ ), percent days with device usage during the 360 days $(82.5 \pm 16.7$ vs. $85.4 \pm 17.7 \%$ ), cumulative device usage hours during the 360 days (1621.2 \pm 510.1 vs. $1727.1 \pm 619.3$ hours), average device usage hours per day during the 360 days ( $4.5 \pm 1.4$ vs. $4.8 \pm 1.7$ hours), average device usage hours per day during the days of device used of this period ( $5.4 \pm 1.0$ vs. $5.5 \pm 1.4$ hours), and percent of days with device usage hours $\geq 4$ hours $(66.0 \pm 21.8$ vs. $68.0 \pm 24.4 \%$ ) between groups during the 360 days after starting CPAP (Table 3).

Table 3. Efficacy and adherence during 360 days after CPAP titration.

\begin{tabular}{|c|c|c|c|}
\hline & $\mathrm{fnPT}(\mathrm{n}=46)$ & $\mathrm{aPT}(\mathrm{n}=22)$ & $p$ value \\
\hline Mean residual AHI* & $3.6 \pm 2.5$ & $3.1 \pm 1.2$ & .718 \\
\hline $\begin{array}{l}\text { Percent days with device } \\
\text { usage (\%) }\end{array}$ & $82.5 \pm 16.7$ & $85.4 \pm 17.7$ & .277 \\
\hline $\begin{array}{l}\text { Cumulative device usage } \\
\text { hours during the } 360 \text { days }\end{array}$ & $1621.2 \pm 510.1$ & $1727.1 \pm 619.3$ & .471 \\
\hline $\begin{array}{l}\text { Average device usage hours } \\
\text { per day during the } 360 \text { days }\end{array}$ & $4.5 \pm 1.4$ & $4.8 \pm 1.7$ & .471 \\
\hline $\begin{array}{l}\text { Average device usage hours } \\
\text { per day during the days of } \\
\text { device used of this period }\end{array}$ & $5.4 \pm 1.0$ & $5.5 \pm 1.4$ & .866 \\
\hline $\begin{array}{l}\text { Percent of days with usage } \\
\geq 4 \text { hours (\%) }\end{array}$ & $66.0 \pm 21.8$ & $68.0 \pm 24.4$ & .901 \\
\hline
\end{tabular}

Data presented as mean \pm standard deviation. *Mean residual AHI was calculated by dividing the number of apnea hypopnea by operating hours.

\section{DISCUSSION}

This study showed that aPT, a new modified method of CPAP titration, was as effective as fnPT in that there was no significant difference in the mean residual AHI (less than five) on CPAP and in adherence (more than $70 \%$ days of device usage and more than four hours usage per day) during the 360 days follow-up. In addition, the average hours of recording time of aPT was significantly shorter than that of fnPT. Compared to fnPT, aPT has an advantage in that it took only one and a half hours.

Another advantage of our procedure was chronobiologically appropriate time slot when aPT was performed. In contrast to our method, the previous two studies $(27,28)$ did the titration in the morning, and the one did not describe the time zone of their titration (29). Regarding the chronobiological change in the vigilance level, Lavie and Segal (30) demonstrated that sleep propensity was second highest in the early afternoon (i.e. 2-4 p.m.) by using ultrashort 7-min sleep/ 13-min wake cycle schedule for 
48 hours. In this context, there is a major benefit about aPT since the patients have the titration during the time slot when they were physiologically sleepy.

It has been widely recognized that the mean residual AHI counted by the CPAP device was a good parameter indicating decrease in respiratory events. Therefore we compared the mean residual AHI of the both groups monitored by the CPAP device during 360 day follow-up. The mean residual AHI in two groups was less than five during the 360 days, which means aPT was effective as fnPT. Unfortunately we could not compare this efficacy to the previous studies (27-29), as they were performed before the era when CPAP devices did not have the function of monitoring respiratory events.

It is unarguable that one of the most important aspects of CPAP treatment is to maintain adherence on a long-term basis. In general CPAP use of 4 hours/night on $70 \%$ of nights has been accepted as a clinical and empiric benchmark of CPAP adherence (16). In fact, there are two factors that define the CPAP adherence : one is frequency of CPAP use and the other is the duration of follow-up. When evaluating the CPAP adherence in respect of these two aspects, our patients initiating CPAP treatment after aPT fulfilled the adherence requirement as good as the patients who underwent fnPT. Both of the groups showed over $80 \%$ days with device usage and averagely 4.5 hours usage per day (360 days), and averagely 5.4 hours usage per day (days used) during the 360 days after starting CPAP.

There are several limitations of this study. Firstly, patients were not randomly assigned to aPT or fnPT group due to the retrospective nature. Secondary, in the aPT, 8 out of 22 patients (36.4\%) achieved REM sleep while all of the 46 patients in the fnPT group achieved REM sleep. As AASM criteria recommends that an optimal titration should include supine REM sleep (25), the aPT could not be completely comparable to the fnPT. This might partially explain the higher dropout rates of the aPT group. Thirdly, it still remains unknown how to choose the appropriate patients who are most likely to be adherent to CPAP treatment after aPT. Thus, further prospective randomly allocated studies would be needed.

In conclusion, aPT has an advantage in that it took only one and half hours to be completed at the time zone when people are physiologically sleepy. Most importantly, the optimal pressure setting determined by aPT was as effective as that by fnPT and there was no difference in long-term adherence between the two groups. Thus, aPT could be a practical alternative way of titration with low cost and labor.

\section{ETHICS APPROVAL}

This study was approved by the Institutional Review Board of Kansai Electric Power Medical Research Institute. The study was conducted in according to the principles of the Declaration of Helsinki.

\section{CONFLICTS OF INTEREST/FUNDING}

Both authors do not have any potential conflicts of interest to disclose, and do not receive any financial support.

\section{AUTHOR'S CONTRIBUTIONS}

Keisuke Kido and Naoko Tachibana contributed to the study conception, study design, and data acquisition. Keisuke Kido primarily analyzed the data and did the statistical analyses.
The first draft of the manuscript was written by Keisuke Kido. Naoko Tachibana revised the manuscript for important intellectual content. Both authors have seen and approved the final manuscript.

\section{ACKNOWLEDGEMENTS}

This research would not have been possible without the support of the following people, Kei-ichi Marumoto, RPSGT, Yoko Uozumi, RPSGT, and Ko-ichiro Taniguchi, MD who involved this study.

\section{REFERENCES}

1. Veasey SC, Rosen IM : Obstructive sleep apnea in adults. N Engl J Med 380 : 1442-1449, 2019

2. Lévy P, Kohler M, McNicholas WT, Barbé F, McEvoy RD, Somers VK, Lavie L, Pépin J-L: Obstructive sleep apnoea syndrome. Nat Rev Dis Primers $1: 15015,2015$

3. Greenberg GD, Watson RK, Deptula D : Neuropsychological dysfunction in sleep apnea. Sleep $10: 254-262,1987$

4. Peppard PE, Szklo-Coxe M, Mae Hla K, Young T : Longitudinal association of sleep-related breathing disorder and depression. Arch Intern Med 166 : 1709-1715, 2006

5. Young T, Blustein J, Finn L, Palta M : Sleep-disordered breathing and motor vehicle accidents in a population-based sample of employed adults. Sleep 20 : 608-613, 1997

6. Levy P, Tamisier R, Arnaud C, Monneret D, Baguet JP, Stanke-Labesque F, Dematteis M, Godin-Ribuot D, Ribuot C, Pepin JL: Sleep deprivation, sleep apnea and cardiovascular diseases. Front Biosci 4 : 2007-2021, 2012

7. Heinzer R, Vat S, Marques-Vidal P, Marti-Soler H, Andries D, Tobback N, Mooser V, Preisig M, Malhotra A, Waeber G, Vollenweider P, Tafti M, Haba-Rubio J : Prevalence of sleep-disordered breathing in the general population : the HypnoLaus study. Lancet Respir Med 3 : 310-318, 2015

8. Gottlieb DJ, Yenokyan G, Newman AB, O'Connor GT, Punjabi NM, Quan SF, Redline S, Resnick HE, Tong EK, Diener-West M, Shahar E : Prospective study of obstructive sleep apnea and incident coronary heart disease and heart failure: The Sleep Heart Health Study. Circulation $122: 352-360,2010$

9. Senaratna CV, Perret JL, Lodge CJ, Lowe AJ, Campbell BE, Matheson MC, Hamilton GS, Dharmage SC : Prevalence of obstructive sleep apnea in the general population : A systematic review. Sleep Med Rev $34: 70-81,2017$

10. D'Ambrosio C, Bowman T, Mohsenin V: Quality of life in patients with obstructive sleep apnea : effect of nasal continuous positive airway pressure - a prospective study. Chest $115: 123-129,1999$

11. George CFP : Reduction in motor vehicle collisions following treatment of sleep apnoea with nasal CPAP. Thorax $56: 508-512,2001$

12. Bazzano LA, Khan Z, Reynolds K, He J : Effect of nocturnal nasal continuous positive airway pressure on blood pressure in obstructive sleep apnea. Hypertension 50 : 417-423, 2007

13. Abud R, Salgueiro M, Drake L, Reyes T, Jorquera J, Labarca G : Efficacy of continuous positive airway pressure (CPAP) preventing type 2 diabetes mellitus in patients with obstructive sleep apnea hypopnea syndrome (OSAHS) and insulin resistance : a systematic review and meta-analysis. Sleep Med 62 : 14-21, 2019

14. Drager LF, Bortolotto LA, Figueiredo AC, Krieger EM, Lorenzi-Filho G : Effects of continuous positive airway 
pressure on early signs of atherosclerosis in obstructive sleep apnea. Am J Respir Crit Care Med 176 : 706-712, 2007

15. Weaver $\mathrm{T}$ : Novel aspects of CPAP treatment and interventions to improve CPAP adherence. J Clin Med 8: 22202227, 2019

16. Kribbs NB, Pack AI, Kline LR, Smith PL, Schwartz AR, Schubert NM, Redline S, Henry JN, Getsy JE, Dinges DF : Objective measurement of patterns of nasal CPAP use by patients with obstructive sleep apnea. Am Rev Respir Dis $147: 887-895,1993$

17. Yang MC, Lin CY, Lan CC, Huang CY, Huang YC, Lim CS, Liu YC, Wu YK: Factors affecting CPAP acceptance in elderly patients with obstructive sleep apnea in Taiwan. Respir Care 58: 1504-1513, 2013

18. Tokunaga T, Ninomiya T, Kato Y, Ito Y, Takabayashi T, Tokuriki M, Sunaga H, Fujieda S : Long-term compliance with nasal continuous positive airway pressure therapy for sleep apnea syndrome in an otorhinolaryngological office. Eur Arch Otorhinolaryngol 270 : 2267-2273, 2013

19. Wang Y, Gao W, Sun M, Chen B : Adherence to continuous positive airway pressure in patients with obstructive sleep apnea in a Chinese population. Respir Care $57: 238-243$, 2011

20. Tan B, Tan A, Chan YH, Mok Y, Wong HS, Hsu PP : Adherence to continuous positive airway pressure therapy in Singaporean patients with obstructive sleep apnea. Am J Otolaryngol 39 : 501-506, 2018

21. McEvoy RD, Antic NA, Heeley E, Luo Y, Ou Q, Zhang X, Mediano O, Chen R, Drager LF, Liu Z, Chen G, Du B, McArdle N, Mukherjee S, Tripathi M, Billot L, Li Q, Lorenzi-Filho G, Barbe F, Redline S, Wang J, Arima H, Neal B, White DP, Grunstein RR, Zhong N, Anderson CS : CPAP for prevention of cardiovascular events in obstructive sleep apnea. N Engl J Med 375 : 919-931, 2016

22. Barbe F, Duran Cantolla J, Sanchez de la Torre M, Carmona C, Barcelo A, Chiner E, Masa JF, Gonzalez M, Marin JM,
Garcia-Rio F, De Atauri JD, Teran J, Mayos M, De la Pena M, Monasterio C, Del Campo F, Montserrat JM : Pressure on the incidence of hypertension and cardiovascular events in nonsleepy patients with obstructive sleep apnea. JAMA $307: 2161-2168,2012$

23. Mehrtash M, Bakker JP, Ayas N : Predictors of continuous positive airway pressure adherence in patients with obstructive sleep apnea. Lung $197: 115-121,2019$

24. Shapiro GK, Shapiro CM : Factors that influence CPAP adherence : an overview. Sleep Breath $14: 323-335,2010$

25. Positive airway pressure titration task force of the American Academy of Sleep Medicine : Clinical guidelines for the manual titration of positive airway pressure in patients with obstructive sleep apnea. J Clin Sleep Med 4 : 157-171, 2008

26. Berry RB, Brooks R, Gamaldo CE, Harding SM, Lloyd RM, Quan SF, MD, Troester MT, Vaughn BV : The AASM Manual for the scoring of sleep and associated events : Rules, terminology and technical specifications. version 2.4. American Academy of Sleep Medicine, Darien, IL, 2017

27. Rosenthal L, Nykamp K, Guido P, Syron ML, Day R, Rice FM, Roth T : Daytime CPAP titration. Chest 114 : 1056 1060, 1998

28. Lloberes P, Rodríguez B, Roca A, Teresa Sagales M, Dolores de la Calzada M, Giménez S, Romero O, Sampol G : Comparison of conventional nighttime with automatic or manual daytime CPAP titration in unselected sleep apnea patients : study of the usefulness of daytime titration studies. Respir Med 98:619-625, 2004

29. Rudkowski JC, Verschelden P, Kimoff RJ : Efficacy of daytime continuous positive airway pressure titration in severe obstructive sleep apnoea. Eur Respir J 18 : 535-541, 2001

30. Lavie P, Segal S: Twenty-four-hour structure of sleepiness in morning and evening persons investigated by ultrashort sleep-wake cycle. Sleep $12: 522-528,1989$ 